A N N A L E S Annales de Bretagne et des Pays de l'Ouest

\title{
Barbaricum comme devoir et défi du souverain chrétien
}

\section{Wojciech Falkowski}

\section{(2) OpenEdition}

Journals

\section{Édition électronique}

URL : http://journals.openedition.org/abpo/1265

DOI : $10.4000 / a b p o .1265$

ISBN : 978-2-7535-1495-9

ISSN : 2108-6443

Éditeur

Presses universitaires de Rennes

Édition imprimée

Date de publication : 20 septembre 2004

Pagination : 407-415

ISBN : 978-2-7535-0053-2

ISSN : 0399-0826

\section{Référence électronique}

Wojciech Falkowski, «Barbaricum comme devoir et défı du souverain chrétien », Annales de Bretagne et des Pays de l'Ouest [En ligne], 111-3| 2004, mis en ligne le 20 septembre 2006, consulté le 19 avril 2019. URL : http://journals.openedition.org/abpo/1265 ; DOI : 10.4000/abpo.1265 


\title{
Barbaricum comme devoir et défi du souverain chrétien
}

\author{
Wojciech FALKOWSKI \\ Professeur à l'Université de Varsovie
}

L'étude des idées d'Alcuin sur les responsabilités d'un monarque chrétien nous amène à analyser deux exemples qui datent d'avant l'arrivée du savant à la cour carolingienne. Après son accession au pouvoir autonome, Charlemagne reçut, vers 775 , une longue lettre de la part d'un prêtre lettré, Cathwulf, qui lui adressa des préceptes et des suggestions quant à la manière d'exercer la royauté. Cette lettre constitue en fait un traité, bien structuré et succinct, sur les obligations du roi, sur les objectifs qu'il devait atteindre et sur les responsabilités qu'il devait assumer. Présenté dans cette lettre en tant que vicaire de Dieu, Charlemagne est investi de la mission de gouverner toute l'Europe. Le rôle du clergé est celui d'un auxiliaire - les évêques, nommés vicaires du Christ, n'ayant leur place qu'au second rang ${ }^{1}$. Selon cette conception, c'est au souverain que revenait la plus haute et indivisible responsabilité de réaliser le plan du Créateur.

Ce fragment, largement commenté ${ }^{2}$, n'est pourtant qu'une partie des longues énonciations qui contiennent aussi bien une brève récapitulation de l'histoire du règne de Charlemagne qu'un ensemble des préceptes et des indications sur la manière d'exercer la royauté. Leur structure est symétrique. Cathwulf réunit d'une part huit événements historiques témoignant de la grandeur du roi des Francs et de l'autre, huit préceptes sur les moyens de remplir le rôle qui lui est imparti ${ }^{3}$. Les faits datant d'un passé récent devaient illustrer les mérites du roi et de la dynastie, ainsi que mettre en évidence l'aptitude du souverain à assumer la mission qui lui était confiée. S'ouvrant par le sacre royal de Charles qui voit se confier l'État des Francs et qui accède au pouvoir sans répandre le sang, l'énumération se termine

1. Epistolae variorum ..., $\mathrm{n}^{\circ} 7$, p. 503 :...quod ipse [Deus] te exaltavit in honorem glorie regni Europae. [...] Memor esto ergo semper, rex mi, Dei regis tui cum timore et amore, quod tu es in vice illius super omnia membra eius custodire et regere, et rationem reddere in die iudicii, etiam per te. Et episcopus est in secundo loco, in vice Christi tantum est.

2. Cf. W. Ullmann, The Carolingian Renaissance..., p. 49-50; W. Ullmann, The Growth of Papal Government..., p. 106.

3. Cf. l'excellente comparaison faite par J. STORY, "Cathwulf... ", p. 10. 
par la description de la campagne victorieuse contre les Lombards, la prise de Pavie et l'entrée dans Rome. La conquête triomphale de l'Italie est le couronnement de la politique menée jusqu'à ce moment-là et reste un signe visible de la grâce dont bénéficiait la dynastie. Dans la conclusion, l'État de Charlemagne est assimilé au territoire du continent tout entier, l'Europe étant traitée par Cathwulf de royaume de Charlemagne, ce dernier ayant obtenu sa position par la volonté de Dieu et à l'issue d'une réalisation qui s'avère la conséquence des phases successives de la domination sur le monde chrétien et sur le peuple qui habita ce territoire rendu au prince par Dieu. Car il était évident que nul roi ne pouvait porter son titre s'il ne régnait pas sur un peuple ${ }^{4}$. La condition pour accéder au pouvoir suprême est d'observer des règles morales fondamentales et d'appliquer des méthodes de bon gouvernement, ce qui est explicité par l'auteur de la lettre dans huit " colonnes".

Ainsi, la mission royale devait reposer sur les principes par lesquels le souverain devait se laisser guider : attester la vérité dans les décisions concernant l'État; faire constamment preuve de patience, de générosité, de force de conviction; réparer le mal et récompenser le bien, alléger les taxes payées par des sujets; assurer à tous des jugements justes devant les tribunaux ${ }^{5}$. C'est donc la liste des vertus du roi qui définissait les objectifs du règne et précisait les moyens de les réaliser. Il est à noter que l'ordre idéal du monde, le modèle de chrétienté préconisé par Cathwulf est mis en rapport étroit avec les actions concrètes du monarque. La partie historique et la vision du règne idéal sont présentées de manière à se compléter l'une l'autre, pendant que la théorie de la gouvernance avait pour source des expériences antérieures de Charlemagne. Un "bref manuel » destiné au roi constitue en fait la suite de la relation du chroniqueur sur les premières années du règne. Des succès obtenus au début du règne sont ainsi liés aux qualités personnelles du souverain qui s'approche de l'idéal et qui est capable de remplir la volonté de Dieu. Observer les règles prescrites devait assurer la bénédiction à Charlemagne et à sa dynastie, et garantir la paix et la prospérité aux gouvernés. La domination sur toute l'Europe conçue comme regnum Europae est donc d'une part le résultat des succès d'une politique menée auparavant et le signe de la grâce divine, et d'autre part, l'obligation d'assumer les devoirs tout particuliers et de se conduire conformément aux règles. C'est dans ce contexte qu'apparaît le personnage de Moïse, vainqueur des Amalécites ${ }^{6}$, ainsi que législateur et juge suprême. L'allusion est transparente - Charlemagne, comme Moïse, réunit les qualités d'un grand chef, qui mène son peuple à combattre des ennemis, et d'un dirigeant ayant la responsabilité de toute la christianitas.

4. Epistolae variorum..., p. 503 : Ergo considerate inter vos diligenter legem Dei constituere super populum Dei...

5. Ibid. J. STORY, "Cathwulf... ", a souligné, à juste titre, signification symbolique du chiffre huit. Un parallèle avec les sept colonnes de la sagesse ne nous semble pas justifié.

6. Ex 17, 9-16. 
Cathwulf n'est pas le seul à se servir d'une telle argumentation. De la même période date la lettre d'un moine lettré, Clément, adressée à un rival puissant des Carolingiens, prince de Bavière, Tassilon ${ }^{7}$. Pleines d'érudition, ses énonciations abondent en références à l'Ancien Testament et établissent toute une liste de comparaisons entre le souverain, d'un côté, et les prophètes et rois bibliques, de l'autre ${ }^{8}$. Dans le message qu'elle porte, la lettre sollicite la victoire du souverain et indique les analogies bibliques qui se rapportent aux expéditions militaires. L'auteur demande pour le prince la force de Samson, après avoir rappelé les victoires de Gédéon du Livre des Juges et la gloire de Jonathan, fils de Saül. Tassilon se voit également comparé à l'empereur Constantin, dont la victoire remportée dans une bataille fut annoncée par une croix de feu apparue sur le ciel. La liste des analogies s'ouvre par l'évocation de l'histoire de Moïse qui, par sa prière, avait obtenu l'aide de Dieu dans le combat contre les Amalécites et, plus tôt, avait fait traverser la mer Rouge à son peuple. L'œuvre de Moïse illustre la protection de Dieu, la puissance de la chrétienté et la responsabilité d'un souverain envers ses sujets. Les correspondances établies équivalaient à la légitimation des conquêtes du prince de Bavière et à la valorisation des succès de ce dernier sur la scène politique. Il se voit présenté comme chef militaire vainqueur et comme l'espoir des chrétiens dans la lutte contre des peuples païens qui encerclaient le peuple élu. La lettre contient des éloges recherchés et des invocations pathétiques qui, par leur ton, ressemblent aux laudes du roi où la prospérité du monarque fait l'objet de prières. Elle diffère du traité de Cathwulf par l'absence de l'évocation des réussites du souverain et par le manque de préceptes sur la manière de gouverner. Cependant, dans les deux cas, on trouve la description de la mission d'un souverain chrétien idéal. M. Garrison a récemment présenté d'une manière très convaincante les analogies de deux textes et l'importance des conseils donnés aux princes ${ }^{9}$.

De nombreuses références au personnage de Moïse en tant que guide et sauveur présentaient ce dernier comme un modèle à suivre et suggéraient que son grand successeur avait fait son apparition. Cela indique le besoin d'un modèle de monarque qui soit en mesure de protéger son peuple, d'organiser l'État, de s'opposer aux ennemis et qui, surtout, jouisse de la grâce de Dieu.

Dans un écrit ultérieur, Alcuin nomme Moïse dux et legista Israhelitici populi ${ }^{10}$, formule qui résume l'idéologie de cet exemple. Il s'agissait du rôle

7. Sur Tassilon et Bavière carolingienne, cf. l'étude classique de H. WOLFRAM, « Das Fürstentum Tassilos... ", ainsi que les travaux plus récents de M. BECHER, Eid und Herrschaft..." ", et de W. Brown, Unjust seizure.

8. Epistolae variorum..., $\mathrm{n}^{\circ}$ 1, p. 496.

9. M. GARRISON, "Letters to a king... ».

10. Alc. Ep. 69, p. 113. La date de la lettre n'est pas établie, l'éditeur indique les années 789 à 796. Dans son remarquable opus magnum et posthumum, D. Bullough, Alcuin : Achievement and Reputation..., p. 370-371, suggère que le destinataire de cette lettre pouvait être le prince Gerold, frère de la reine Hildegarde, qui remplaça le duc Tassilon. La 
à la fois de chef et de guide, et de législateur, du rôle à la fois d'un dirigeant conscient de ses responsabilités et d'un organisateur avisé. Moïse inaugura une nouvelle ère de la Loi et de l'espérance. Le nouvel ordre qui fut établi reposait sur la Loi obtenue de Yahvé. Il s'ensuivit un nouvel ordre social qui plaisait à Dieu et qui fut la base de l'organisation de l'État. Pour Alcuin, il n'y a pas de doute que la période de grâce, commencée avec Moïse, dure toujours avec les lois établies d'abord par lui et ensuite ses succeseurs ${ }^{11}$. Le besoin d'avoir un grand souverain devient ainsi d'autant plus évident.

Dans le dialogue qu'il entretient avec Charlemagne pendant les derniers préparatifs concernant l'expédition à Rome durant été $800^{12}$, le conseiller savant reprend les exemples du livre de l'Exode. La victoire remportée sur les Amalécites lui sert, comme auparavant, à mettre en exergue le rôle prépondérant du nouveau Moïse. D'un autre côté, la lettre commence par un appel à poursuivre la conquête triomphale des peuples barbares et païens ${ }^{13}$ :

"Que Dieu fasse que les victoires de Votre Majesté inspirent la peur aux peuples ennemis. Que l'on croie seulement en notre Dieu et Seigneur, Jésus Christ, et qu'il soit le seul à être aimé et vénéré. "

Tandis que c'est la puissance qui permet d'accéder à la royauté et de protéger les sujets, des efforts soutenus en vue de propager la foi chrétienne font d'un souverain un prédicateur. Ceci permet au monarque d'atteindre une double perfection : tout en détenant le pouvoir sur terre, il bénéficie d'une sagesse spirituelle.

Dans des analogies puisées dans la Bible, l'enseignement et les prophéties d'Élie égalaient les accomplissements de Moïse. Au grand législateur succédait un prédicateur illustre. Charlemagne devait s'inspirer de ces deux exemples ${ }^{14}$. Ainsi, l'instruction du peuple est placée au même niveau que les talents d'un chef militaire et d'un organisateur. Les analogies bibliques laissaient entendre que le souverain restait en relation directe avec Dieu, à l'instar des prophètes qui recevaient des dispositions et des signes directement du Créateur. Les figures de Moïse et d'Élie n'étaient donc pas uni-

deuxième remarque concerne le déchiffrage du mot legista qu'il propose lire comme legifer, ce qui ne change pas notre interprétation.

11. Alc. Ep. 143, p. 226 : Regnavit mors ab Adam usque ad Moysen; et significant : usque ad ultima tempora legis, quae per Moysen data est et decucurrit in Christi dei nostri tempora; Alc. Ep. 307, p. 466 et p. 467 : formulations similaires. D. Bullough, Alcuin : Achievement and Reputation..., p. 267, n. 44 suppose que cette dernière lettre provient des années 802/803.

12. D. A. Bullough, Alcuin : Achievement and Reputation..., p. 64 et n. 154, date cette lettre avant la fin d'août 799 .

13. Alc. Ep. 178, p. 294 : Hoc enim faciat Deus et hoc addat, ut triumpho terroris vestri inimicas undique subiciat gentes; et suavissimo suae dilectionis iugo in christiana fide ferocissimos subiciat animos, ut solus deus et dominus noster Iesus Christus credatur, colatur atque ametur.

14. Alc. Ep. 143, p. 226. 
quement des modèles à suivre et des personnages porteurs des règles que le monarque carolingien devait observer dans son activité et dans sa manière d'agir. Par leur évocation, on suggérait que le monarque était chargé de mettre en œuvre un plan divin. Les rois et les prophètes de l'Ancien Testament réunissent toutes les qualités d'un souverain idéal qui doit être à la fois un illustre chef et guerrier, un juste législateur et juge, un maître plein de sagesse et un visionnaire. Le raisonnement d'Alcuin est donc marqué par l'esprit de suite et se réfère à des modèles anciens bien connus où le souverain se voyait obligé de joindre à sa vocation de chef militaire celle de propagateur de la foi chrétienne. Il est évident que le postulat de l'élargissement du territoire de la chrétienté faisait partie du projet royal ${ }^{15}$. Même si toute action militaire était soumise aux règles morales, elle apportait aussi une preuve indispensable des talents de chef et des capacités du souverain d'appliquer des principes religieux et éthiques. Des conseils et des opinions exprimées dans la correspondance adressée à Charlemagne ne sont pas des commentaires théoriques sur un État idéal et sur son souverain, mais des remarques pratiques sur les moyens d'accomplir la mission de roi ${ }^{16}$.

Dans la conception d'Alcuin, la vision de dilatatio imperii christiani allait de pair avec la mise en exergue des responsabilités du monarque - ce qui, finalement, prit la forme d'un programme succinct de gouvernement d'un souverain illustre. Dans sa lettre qui date de l'été 799, Alcuin commente avec soulagement l'achèvement de la campagne contre les Saxons pour passer immédiatement à l'établissement d'une liste de dix obligations fondamentales du roi ${ }^{17}$. Une étroite complémentarité de ces devoirs aboutit à une vision cohérente. La liste commence par un appel à gouverner l'État constamment et sans répit, ainsi qu'à veiller sur tout sur place. Les recommandations de iter agere et regna gubernare s'appliquaient à la pratique quotidienne du gouvernement et menaient à d'autres dispositions qui se rapportaient à l'exercice de la justice, au pouvoir de légiférer, à la protection des églises, des pèlerins et des défavorisés, ainsi qu'à l'instruction du peuple. L'ensemble se termine par l'exhortation à indiquer à tous les sujets le chemin de la vie - via vitae. Alcuin, dans cette liste d'instructions, imita l'exemple du Pseudo-Cyprien, qui avait établi un relevé des devoirs d'un bon souverain ${ }^{18}$. Cependant, il faut souligner que le conseiller de Charlemagne accentua l'aspect pratique des conseils formulés dans sa lettre et s'avérait plus préoccupé de peser sur la Realpolitik que de proposer un miroir du prince idéal (speculum principis) ${ }^{19}$. Le souverain doit

15. Alc. Ep. 202, p. 336; Alc. Ep.185, p. 310.

16. Nous partageons l'argumentation de J. M. WALlACE-HaDRILL, Early Germanic Kingship..., p. 101, selon laquelle le roi doit être en même temps le doctor et le dux.

17. Alc. Ep. 177, p. 293.

18. Cf. L. Wallach, Alcuin and Charlemagne..., p. 10. L'influence de Pseudo-Cyprien est analysée par I Deug Su, Cultura e Ideologia..., p. 32-34.

19. C'est la suggestion de L. Wallach concernant au premier rang le traité De rhetorica, mais il exprime la même opinion basée également sur les autres travaux d'Alcuin, $o p$. cit., p. $9-13$, p. 32 , p. 229 . 
guider son peuple vers le salut éternel et accomplir sa mission royale à l'instar des prophètes et des rois bibliques. La royauté est un chemin à parcourir par le souverain lui-même et par ses sujets, conformément aux vérités de la foi et aux préceptes donnés par les conseillers, un chemin comparable au passage de la mer Rouge par le peuple d'Israël, mené par Moïse. Ainsi, la via vitae devenait via regia, alors que le roi se chargeait du rôle de guide et d'auxiliaire de tous les chrétiens ${ }^{20}$.

Le titre de rector, attribué à Charlemagne à côté du titre de roi dans l'Admonitio generalis, définit les tâches du souverain d'une manière nouvelle et complémentaire. Il y apporte une signification supplémentaire qui, auparavant, ne se rapportait pas à la notion de rex, telle qu'on la considérait généralement. Le capitulaire de 789 propose une formule qui, d'une façon novatrice, décrit les obligations du souverain chrétien ${ }^{21}$. Dans la titulature composée des deux désignations : rex et rector, les deux termes se complètent pour préciser le contenu de la mission dont était investi le souverain du royaume des Francs ${ }^{22}$. Le premier terme se rapporte au postulat regna gubernare, l'autre à celui de populum corrigere. Ainsi le terme de rex se réfère à l'exemple de Moïse tandis que la tradition de prédication est symbolisée par le prophète Élie. La théologie politique créée par Alcuin apportait une modification significative par rapport aux conceptions antérieures. Tout en gouvernant l'État et protégeant l'Église, le souverain devenait responsable du salut éternel de ses sujets et devait leur servir de guide et de maître. Cette vision du gouvernement s'avère la mise en œuvre exacte du plan divin. La fonction de rector ne se limitait donc pas aux affaires de l'Église ou aux matières religieuses ${ }^{23}$. Certes, elle ne signifiait pas rector ecclesiae, désignant le dirigeant d'un État et des sujets, ainsi qu'un souverain qui réalisait un programme politique bien défini ${ }^{24}$.

La lettre 177, mentionnée plus haut, se termine par un hymne élogieux où Dieu est remercié d'avoir donné à l'univers un guide (rector) aussi remarquable. Les talents militaires y sont juxtaposés aux efforts visant à assurer aux sujets le salut éternel alors que l'activité de prédicateur et de maître est mentionnée à côté des triomphes militaires. L'exhortation à assurer la sécurité comporte également un appel à entreprendre des actions assurant le salut éternel de tous. Le consilium pacis est donc en même temps le consilium salutis, le roi demeurant un meilleur guide dans les deux cas. Le grand guerrier est aussi un responsable visionnaire qui entretient des relations privilégiées avec le Créateur. C'est pour cela qu'Alcuin formule le sou-

20. Cf. les remarques de J. M. WAllace-Hadrill, " The Via Regia... ", p. 22-32.

21. Capitularia $1, \mathrm{n}^{\circ} 22$.

22. L'intitulation initiale de l'acte est la suivante : Ego Karolus, gratia Dei eiusque misericordia donante rex et rector regni Francorum et devotus sanctae aecclesiae defensor humilisque adiutor... Sur Alcuin comme l'auteur de l'Admonitio generalis, cf. F.-C. ScHEIBE, "Alcuin und die Admonitio generalis".

23. Voir H. WolfRAM, Intitulatio, tome I, p. 239-242.

24. Nous ne sommes pas d'accord avec l'analyse d'E. MAGNOU-NORTIER, "Charlemagne... ", p. 363, qui considère cette titulature comme bizarre et composée un demi-siècle plus tard. 
hait que Dieu lui donne la sagesse dans ses décisions devant mener au salut éternel de tous les peuples du royaume d'Europe - Det tibi consilium pacis simul atque salutis ${ }^{25}$.

Le titre de rector mentionné au début du capitulaire connu sous le nom d'Admonitio generalis revient à maintes reprises dans la correspondance d'Alcuin. Il devient la désignation indispensable dans la description des règles de l'exercice du pouvoir royal en élargissant l'étendue des obligations du roi et en marquant le changement des normes jusqu'alors à l'honneur. Cette notion était présente aussi dans le contexte de l'instruction des sujets et de la conquête réussie des peuples païens. Dans une lettre dont la datation reste incertaine, écrite probablement dans les années 794-795, Alcuin présente, en se prononçant au nom de tous les habitants du royaume, un éloge exhaustif du roi des Francs. Y sont juxtaposés les titres de " guide" (rector) et de prédicateur (praedicator). Comme attributs du pouvoir détenu apparaissent le glaive tenu à la main droite et la trompette convoquant les sujets à écouter des leçons de foi ${ }^{26}$. Le roi David auquel Charlemagne était si souvent comparé, y est représenté comme un chef victorieux qui conquiert des peuples pour leur enseigner, déjà dans son rôle de prédicateur, les lois de Dieu. Le gouvernement d'un guide illustre assurait aux chrétiens la vie en paix, les peuples païens étant retenus par la peur. Ce modèle sert donc le souverain qui réunit les capacités d'un chef, les talents d'un prédicateur et d'un maître, ainsi que le savoir d'un législateur. Ceci devient la condition de l'accomplissement de la mission qui lui est confiée par Dieu et de la capacité d'assurer le bonheur des sujets. Il n'est pas fortuit qu'un capitulaire de programme (pour utiliser l'expression de F.-L. Ganshof), l'Admonitio generalis, insiste que les missi qui, s'appuyant sur l'autorité du roi, parcourent l'État et corrigent ce qui est à corriger (corrigerent quae corrigenda sunt ${ }^{27}$ ). Le couronnement impérial n'a rien changé à cette distribution des responsabilités. Dans l'exercice de la nouvelle dignité, le rector reste un complément indispensable, comme le prouve la titulature utilisée dans la lettre de 802 (imperator invicte et sapientissime rector $^{28}$ ). Notons que la couronnement impérial ne changea rien à la titulature ni à la terminologie idéologique du discours tenu par le conseiller et son souverain. Dans cette lettre, Charlemagne est toujours intitulé rex, même si l'auteur ajoute les désignations traditionnellement réservées uniquement aux empereurs ${ }^{29}$. Le titre royal était entièrement suffisant pour désigner la mission du prince idéal et le programme de Charlemagne. D. Bullough avait raison de souligner que le pouvoir terrestre provenait de l'autorité du Christ et, dans ce contexte, l'échange du titre royal contre un titre impérial n'était pas une chose fondamentale ${ }^{30}$.

25. Alc. Ep. 177, p. 293.

26. Alc. Ep. 41, p. 84.

27. Capitularia 1, p. 53.

28. Alc. Ep. 257, p. 415.

29. Ibid. : O rex augusto clarissime dignus honore, [...] decus imperii.

30. D. A. Bullough, Alcuin : Achievement and Reputation, p. 407. 
Dans la théologie politique d'Alcuin, le terme de rector devient une notion clé qui apporte la preuve que l'exercice du pouvoir n'était limité ni juridiquement, ni politiquement. Alors qu'il n'y avait pas de frontières territoriales pour la réalisation de la mission du souverain chrétien, les devoirs et les droits restaient à la mesure des responsabilités acceptées par le roi des Francs. Dans son éloge de l'œuvre de Charlemagne, Alcuin le qualifie de " guide et défenseur du peuple chrétien; pieux, avisé et juste, réparant le mal et consolidant la vertu, il use de toutes ses forces pour propager la sainteté et répandre le nom de Dieu dans tous les recoins de la terre ${ }^{31}$ ". Ainsi, la lumière de la foi catholique pénètre jusqu'aux confins de la terre. En sa qualité de rector, le roi des Francs doit instruire tous les peuples et leur enseigner la vraie foi. Alcuin affirme nettement que Charlemagne devait mener son action au-delà des frontières de l'empire et qu'il pouvait et devait recourir à la force afin d'atteindre les objectifs qui lui étaient assignés. Le glaive du guerrier et le pupitre du prédicateur deviennent les attributs du souverain qui s'en sert afin d'accomplir sa mission, aussi bien dans le royaume que sur les territoires situés au-delà du limes.

Cependant, ce programme ne se rapporte qu'à Charlemagne, qui bénéficiait de la position la plus haute et la plus privilégiée parmi tous les monarques. Des conseils sur la manière de gouverner relèvent du débat sur la mission du roi des Francs, mais ne constituent pas pour autant un ensemble des préceptes pour un monarque idéal. Charlemagne n'avait nul besoin de speculum principis $^{32}$, lui-même étant un modèle à suivre. En tant que rector populi christiani ${ }^{33}$, il n'était pas un souverain ordinaire ni l'un de ces nombreux gouverneurs qui administraient une partie du continent. Il échappait aux jugements de valeur qui s'appliquaient aux rois amis qui gouvernaient en Northumbrie ou en Mercie, et n'avait pas besoin d'obtenir des instructions de la part de ses conseillers sur la politique qu'il devait choisir (son fils Pépin reçut de telles instructions ${ }^{34}$ ). Il ne devait pas non plus faire reconnaître son autorité, car il l'obtenait par la volonté de Dieu. Cette mission s'étendait sur le monde de l'époque (orbis terrarum); pour Alcuin et pour Charles, l'imperium christianum n'était ni une notion politique dans le sens des frontières établies, ni religieuse dans le sens d'un territoire habité et gouverné par les chrétiens, mais une notion idéologique en tant qu'espace accessible au roi des Francs, à ses armées et à son enseignement. L'imperium christianum relevait de la personne du souverain et du pro-

31. Alc. Ep. 121, p. 176 : ...pium, prudentem et iustum [...] rectorem et defensorem; qui prava corrigere et recta corroborare et sancta sublimare omni intentione studeat, et nomen domini Deiexcelsi per multa terrarum spatia dilatare gaudeat. .

32. Nous partageons l'opinion de H. H. ANTON, Fürstenspiegel und Herrscherethos..., p. 87-90 dans sa polémique avec L. Wallach.

33. Alc. Ep. 174, p. 288.

34. Cf. Alc. Ep. 119, qui consiste entièrement en conseils donnés au jeune roi d'Italie par un proche collaborateur de Charlemagne pour lui indiquer comment gouverner, quelles méthodes utiliser et quels buts poursuivre dans son royaume. Parmi d'autres : Esto fortis in adversarios, fidelis in amicos, humilis christianis, terribilis paganis... 
gramme qu'il réalisait et non pas d'un territoire donné, délimité par des frontières bien gardées et des zones frontalières ${ }^{35}$. Le limes Saxonicus et le limes Souabicus ne désignaient que temporellement l'étendue de l'État du roi des Francs, qui devait gagner des terrains nouveaux en tant que regnum Europae de Charlemagne et qu'espace à recevoir une seule et vraie foi ${ }^{36}$.

\section{RESUME}

Les obligations d'un souverain chrétien ne se limitaient pas à son État ni au peuple à lui confié par Dieu. Dans ses lettres, en reprenant l'idéologie déjà existante (analysées ici d'après l'exemple des lettres de Catwulf et Clément), Alcuin souligna les devoirs de Charlemagne vis-à-vis de tout le continent européen. Sa mission s'étendait sur le monde de l'époque, parce que l'empire chrétien était au premier rang une notion idéologique en tant qu'espace accessible au roi des Francs, à ses armées et à son enseignement.

\section{ABSTRACT}

The duties of a Christian sovereign were not limited to its State and to the people given to him by God. Alcuin, in his letters, using the old ideology already existing (examined on the basis of Cathwulf's and Clemens' letters) stressed Charlemagne's duties concerning all Europe. The mission of the King was to be implemented in all the world of that time since the Christian empire was rather an ideological term in the meaning of the space with open access to the king of the Franks, his army and teaching.

35. Cf. les réflexions sur la nature des frontières à l'époque carolingienne dans l'article de T. Noble, "Louis the Pious and the Frontiers... », p. 333- 339, et dans J. Smith, " Fines imperii...".

36. Ces remarques nous conduisent à la problématique de l'Église comme altera respublica et des limites de son influence, voir J. L. NELSON, " On the limits of the Carolingian Renaissance". 
